Check for updates

Cite this: Phys. Chem. Chem. Phys., 2020, 22, 2379

Received 9th November 2019,

Accepted 6th January 2020

DOI: $10.1039 / \mathrm{c} 9 \mathrm{cp} 06089 \mathrm{~h}$

rsc.li/pccp

\section{Calculation of vibrationally resolved absorption and fluorescence spectra of the rylenes $\dagger$}

\author{
Jonas Greiner (iD ${ }^{a}$ and Dage Sundholm (D) *b
}

A generating function method was used to simulate the vibrationally resolved absorption and emission spectra of perylene, terrylene and quaterrylene. This method operates on the basis of adiabatic excitation energies and electronic ground and excited state vibrational frequencies. These parameters were calculated using density functional theory with the PBEO functional for perylene and terrylene and with the BH-LYP functional for quaterrylene. The vertical excitation energies of the lower excited states were calculated using functionals with differing amounts of Hartree-Fock exchange. The optimal functional for each molecule was chosen by comparing these energies to literature excitation energies. Using this technique the calculated absorption spectra and the calculated emission spectrum of perylene were found to be in excellent agreement with the literature experimental spectra after introducing a shift and a scaling factor. The most prominent bands of the absorption spectra were assigned to their respective vibronic transitions.

\section{Introduction}

Perylene, terrylene and quaterrylene form a homologous group of polycyclic aromatic hydrocarbons (PAHs), the rylenes. Derivatives of these compounds are well known for their photochemical properties and their consequent usage in dyes and pigments as they exhibit intense and broad visible and infrared light absorption and high quantum yield fluorescence. ${ }^{1}$ Well-known examples are the perylene monoimides and diimides. Modern optical applications such as organic photovoltaic cells, ${ }^{2,3}$ dye lasers ${ }^{4}$ and organic field effect transistors ${ }^{5}$ on the basis of rylene derivatives are also being studied. Therefore it is worth investigating the vibronically excited states of the parent structures. Another important property of the PAHs is their long photostability. ${ }^{6,7}$ It is postulated that these molecules and their cations are common in the interstellar medium $^{8-11}$ and could therefore be the source for part of the diffuse interstellar bands (DIBs). ${ }^{12-15}$ The identification of the molecules that are responsible for the DIBs is possible through the comparison with low-temperature spectra with no solvent effects. Quantum chemical calculations facilitate the prediction of these lowtemperature spectra.

\footnotetext{
${ }^{a}$ Institut für Physikalische Chemie, Johannes Gutenberg-Universität Mainz Duesbergweg 10-14, D-55128 Mainz, Germany.

E-mail: jgreiner@students.uni-mainz.de

${ }^{b}$ Department of Chemistry, University of Helsinki, P.O. Box 55 (A.I. Virtanens plats 1), FIN-00014 University of Helsinki, Finland. E-mail: Dage.Sundholm@helsinki.fi

$\dagger$ Electronic supplementary information (ESI) available: The Cartesian ground and excited state coordinates of the optimized molecular structures, the oscillator strengths of the vertical excitations and the calculated ground and excited state harmonic frequencies of the studied molecules are given as supporting information. All assigned bands in the calculated absorption spectra with a relative intensity of above $2 \%$. See DOI: $10.1039 / \mathrm{c} 9 \mathrm{cp} 06089 \mathrm{~h}$
}

Photon absorption and fluorescence of molecules follow the Franck-Condon principle. The smaller the shift in nuclei positions induced by the electronic excitation, the higher the observed intensity of the transition. ${ }^{16,17}$ In the zero-temperature approximation only the vibronic ground state is populated; excitations can only occur from this state. Most molecules also follow Kasha's rule, i.e., photon emission occurs from the vibrational ground state of the lowest electronically excited state. ${ }^{18}$ Within these approximations, the vibronic structure of the absorption spectrum is only a function of the excited state vibrational spectrum, while the fluorescence spectrum is a function of the ground state vibrational spectrum. The theoretical investigation of the vibronic spectra of molecules is thus possible through the calculation of electronic ground and excited state geometries and vibrational frequencies within the harmonic approximation. The ground state energy and geometry is accessible through density functional theory (DFT) while the time-dependent variant (TD-DFT) is necessary to investigate the electronically excited states. The coupling between vibrational and electronic transitions can be simulated in the harmonic approximation by describing the geometrical and vibrational differences between the ground and excited states through the Duschinsky rotation. ${ }^{19}$ The geometric displacement and the Duschinsky rotation matrix are used to construct a generating function that allows the simulation of absorption and fluorescence spectra within the zerotemperature approximation. This method has been developed and implemented by Tapavicza $e t a l^{20,21}$ The simulated spectra can be compared with ultraviolet-visible (UV-Vis) absorption and fluorescence spectra measured by matrix isolation spectroscopy. Absorption spectra for the perylene, terrylene and quaterrylene molecules have been recorded by Halasinski et al. ${ }^{22}$ The absorption and fluorescence spectra of perylene have been recorded by Joblin $e t a l^{23}$ 
As with all DFT methods the choice of the correct functional is significant for the accuracy of the calculated results. This is especially true for photochemical applications. The amount of 'exact' HF exchange included in the hybrid correlationexchange-functionals has a large influence on the TD-DFT vertical excitation energies and the resulting spectra as can be seen in the literature. ${ }^{24,25}$

\section{Methods}

All calculations were done using Turbomole ${ }^{26-28}$ with the Karlsruhe triple-zeta basis set with double polarization functions (def2-TZVPP) ${ }^{29}$ and the m5 grid. ${ }^{30}$ The functionals used are the TPSSh functional with 10\% HF exchange, ${ }^{31-33}$ the B3-LYP functional with $20 \%$ HF exchange, ${ }^{34-36}$ the PBE0 functional with $25 \% \mathrm{HF}$ exchange $^{31,37,38}$ and the BH-LYP functional with $50 \% \mathrm{HF}$ exchange. $^{34,35,39}$ The symmetry of all molecules is $D_{2 \mathrm{~h}}$ and they are oriented in the $x y$-plane. The ground state geometry was optimized using density functional theory (DFT) ${ }^{40}$ and the respective functionals. The optimized molecular structures are presented in Fig. 1. The vertical excitation energies were calculated from the ground state geometries using timedependent density functional theory (TD-DFT). ${ }^{41-43}$ The excited state geometries were optimized using TD-DFT. ${ }^{40,43,44}$

The vibrational frequencies of the molecules in their ground state were calculated from the analytic second derivative of the energy using the AOFORCE module. ${ }^{47,48}$ The second derivatives of the excited state molecules were calculated numerically from

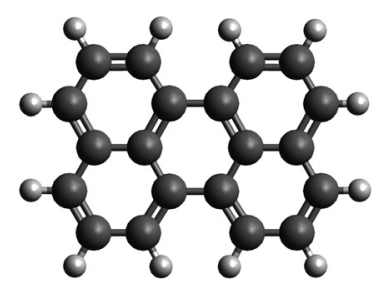

(a) Perylene

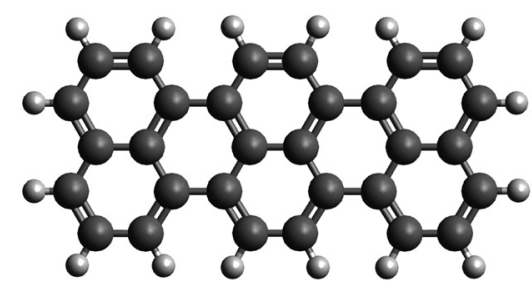

(b) Terrylene

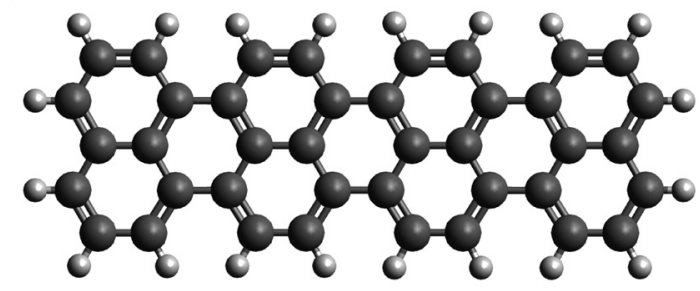

(c) Quaterrylene

Fig. 1 Molecular structures of the studied molecules. The graphics were created using Avogadro. ${ }^{45,46}$ the analytical gradients using the NUMFORCE module. The RADLESS module was used for the simulation of vibronic absorption and fluorescence spectra. ${ }^{20,21}$ The resolution of the calculated spectra can be raised by increasing the integration time. An integration time of 2.42 ps was found to be proficient for the comparison with the experimental spectra. Additionally, lifetime broadening can be employed to permit a visual comparison with the experimental spectra. The lifetime for the spectra of each molecule were chosen to reproduce the spectral widths observed in the respective experimental spectrum. The assignment of bands is easier with very narrow spectral widths. Therefore the absorption bands were assigned to a high-resolution spectrum with a lifetime of $2.42 \mathrm{ps}$ and an integration time of $120.94 \mathrm{ps}$.

\section{Results and discussion}

\subsection{Vertical excitation energies}

To investigate the amount of HF exchange needed for the optimal prediction of the spectra, the vertical excitation energies of the two lowest $\mathrm{B}_{2 \mathrm{u}}$ and $\mathrm{B}_{3 \mathrm{u}}$ states were calculated using the TPSSh, B3-LYP, PBE0 and BH-LYP functionals. These transitions have a non-vanishing transition dipole moment and are therefore experimentally accessible. The excitation energies were calculated on the basis of the optimized ground state structures of the respective functional. The results are displayed in Table 1.

The most accurate energies are obtained using the PBE0 functional (25\% HF exchange) for the perylene and terrylene molecules and using the BH-LYP functional (50\% HF exchange) for the quaterrylene molecule. Therefore these respective functionals should yield the best results for the simulation of the absorption and emission spectra.

\subsection{Absorption spectra}

The functionals determined to accurately reproduce the experimental vertical excitation energies were used to calculate

Table 1 Excitation energies of the two lowest $B_{2 u}$ and $B_{34}$ states of perylene, terrylene and quaterrylene (in eV) calculated at the TPSSh, B3-LYP, $\mathrm{PBEO}$ and $\mathrm{BH}-\mathrm{LYP}$ levels. Experimental excitation energies from the band origin in the neon matrix spectrum are also reported. ${ }^{22}$ The oscillator strengths are given in the ESI

\begin{tabular}{|c|c|c|c|c|c|c|}
\hline \multirow[b]{2}{*}{ Molecule } & \multirow[b]{2}{*}{ State } & \multicolumn{4}{|l|}{ DFT } & \multirow[b]{2}{*}{ Exp. } \\
\hline & & TPPSh & B3-LYP & PBE0 & BH-LYP & \\
\hline \multirow[t]{4}{*}{ Perylene } & $1 B_{3 u}$ & 2.72 & 2.79 & 2.88 & 3.18 & \multirow[t]{4}{*}{2.96} \\
\hline & $1 \mathrm{~B}_{2 \mathrm{u}}$ & 3.92 & 4.00 & 4.14 & 4.51 & \\
\hline & $2 \mathrm{~B}_{2 \mathrm{u}}$ & 4.63 & 4.81 & 5.00 & 5.66 & \\
\hline & $2 \mathrm{~B}_{3 \mathrm{u}}$ & 5.21 & 5.33 & 5.51 & 6.01 & \\
\hline \multirow[t]{4}{*}{ Terrylene } & $1 B_{3 u}$ & 2.07 & 2.15 & 2.22 & 2.50 & \multirow[t]{4}{*}{2.35} \\
\hline & $1 \mathrm{~B}_{2 \mathrm{u}}$ & 3.09 & 3.19 & 3.31 & 3.68 & \\
\hline & $2 \mathrm{~B}_{2 \mathrm{u}}$ & 3.58 & 3.74 & 3.89 & 4.38 & \\
\hline & $2 \mathrm{~B}_{3 \mathrm{u}}$ & 4.00 & 4.21 & 4.40 & 4.97 & \\
\hline \multirow[t]{4}{*}{ Quaterrylene } & $1 \mathrm{~B}_{3 \mathrm{u}}$ & 1.71 & 1.78 & 1.84 & 2.12 & \multirow[t]{4}{*}{2.04} \\
\hline & $1 \mathrm{~B}_{2 \mathrm{u}}$ & 3.15 & 3.29 & 3.43 & 3.86 & \\
\hline & $2 \mathrm{~B}_{3 \mathrm{u}}$ & 3.17 & 3.38 & 3.57 & 4.17 & \\
\hline & $2 B_{2 u}$ & 3.44 & 3.63 & 3.80 & 4.32 & \\
\hline
\end{tabular}


the equilibrium geometry, normal modes and vibrational frequencies of the ground state $S_{0}$ and the first electronically excited state $S_{1}$. The normal modes, vibrational frequencies, transition dipole moments as well as the ground and excited state energies at their respective equilibrium geometries make the calculation of vibronic absorption and fluorescence spectra possible. The calculated absorption spectra can be compared to the $4 \mathrm{~K}$ neon matrix isolation experimental spectra recorded by Halasinski et $a .^{22}$

All calculated absorption spectra are red-shifted compared to the measured spectrum. This difference must be attributed to the calculated adiabatic excitation energies which appear to be underestimated by the TD-DFT methodology. The calculated adiabatic excitation energies for the perylene, terrylene and quaterrylene molecules are $21796 \mathrm{~cm}^{-1}(2.70 \mathrm{eV}), 16887 \mathrm{~cm}^{-1}$ $(2.09 \mathrm{eV})$ and $15667 \mathrm{~cm}^{-1}(1.94 \mathrm{eV})$ respectively. The $0-0$ band of the calculated spectra can be reproduced from the adiabatic excitation energies when the zero-point vibrational energies of the ground and excited states are accounted for. Additionally the experimental spectra of all three molecules include electronic transitions to higher excited states, which can be seen between $35000 \mathrm{~cm}^{-1}$ and $55000 \mathrm{~cm}^{-1}$ in the literature. ${ }^{22}$ These transitions have not been calculated and therefore cannot be compared. The energy intervals between the different bands are higher in the calculated spectra. In the experimental spectrum these intervals decrease with higher energies. The reason for this discrepancy is the usage of the harmonic approximation in the calculation which leads to overestimation of the vibrational energies, because anharmonic effects are not considered. The wavenumbers of the calculated spectrum can be scaled to the experimental spectrum to account for the error introduced by the harmonic approximation. The juxtaposition of the calculated spectra into the experimental spectra measured by Halasinski et $_{\text {al. }}{ }^{22}$ is shown in Fig. 2.

The calculated spectra are shifted by $3380 \mathrm{~cm}^{-1}, 3060 \mathrm{~cm}^{-1}$ and $2660 \mathrm{~cm}^{-1}$ to higher energies and scaled by a factor of 0.98 , 0.99 and 0.93 respectively to make a qualitative comparison of the band structure possible.

When scaled and shifted, the calculated absorption spectrum of perylene in Fig. 2a matches the experimental spectrum precisely. The spectral widths of the experimental bands can be replicated accurately using lifetime broadening. This result underlines the potential of the generating function method to reproduce experimental spectra with high accuracy. Fig. 2a is a testament to the quality of both the experimental and the calculated spectrum. The calculated bands with a relative intensity of above $10 \%$ of the $0-0$ band are reported in Table 2 . The bands were assigned through comparison with the calculated vibrational spectrum of the excited state. Additionally, all bands with a relative intensity of above $2 \%$ of the $0-0$ peak are reported and assigned in the ESI. $\dagger$ Joblin et al. ${ }^{23}$ matched the combination bands in their experimental spectrum to the respective simultaneously excited fundamental bands. For instance, they assigned the band at a shift of $1640 \mathrm{~cm}^{-1}$ to a combination of the bands at $348 \mathrm{~cm}^{-1}$ and $1293 \mathrm{~cm}^{-1}$ and the band at a shift of $1950 \mathrm{~cm}^{-1}$ to a combination of the bands at $348 \mathrm{~cm}^{-1}$

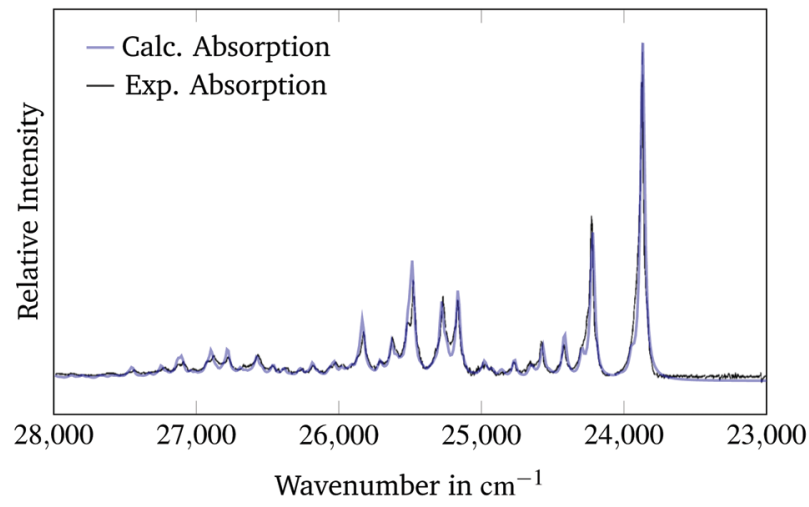

(a) Spectra of perylene, a lifetime parameter of $0.290 \mathrm{ps}$ was used for broadening.

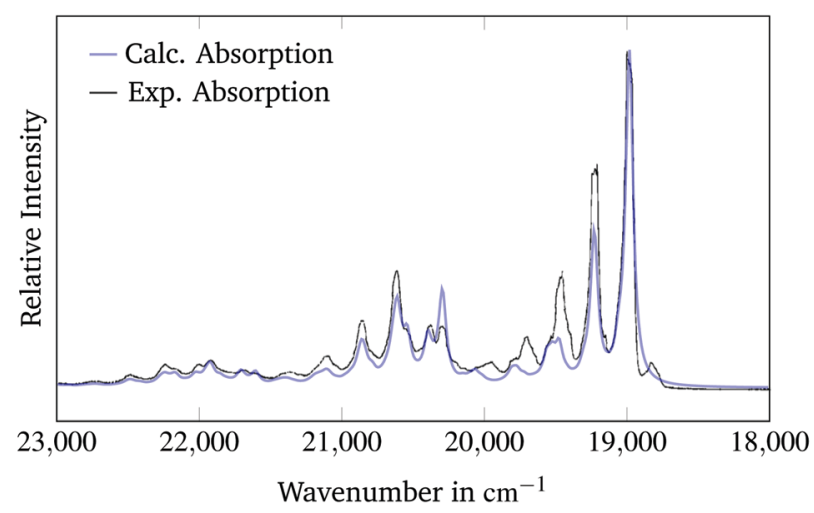

(b) Spectra of terrylene, a lifetime parameter of $0.169 \mathrm{ps}$ was used for broadening.

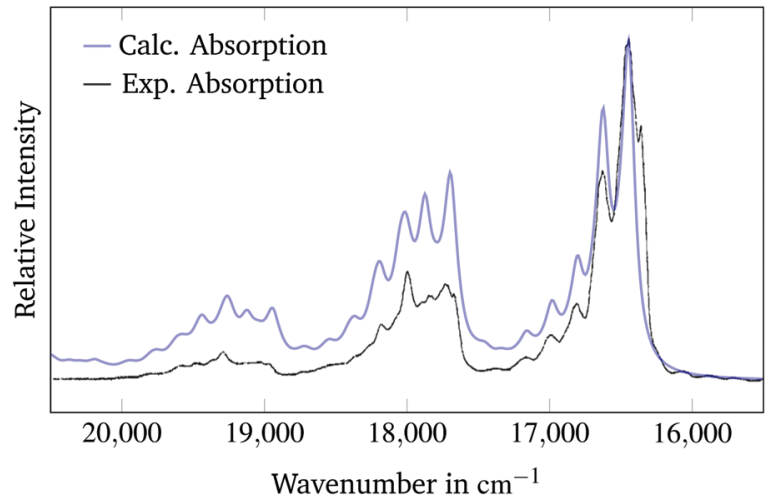

(c) Spectra of quaterrylene, a lifetime parameter of $0.097 \mathrm{ps}$ was used for broadening.

Fig. 2 Calculated and experimental ${ }^{22}$ absorption spectra of the $1 \mathrm{~B}_{3 \mathrm{u}}$ state of perylene, terrylene and quaterrylene. The simulated spectra are scaled and shifted to match the experimental absorption spectra.

and $1601 \mathrm{~cm}^{-1}$. The assignment in Table 2 reaches the same conclusions when accounting for the harmonic approximation.

The comparison of the calculated absorption spectrum of terrylene to the experimental absorption spectrum in Fig. $2 \mathrm{~b}$ shows small differences in the band intensities. All band intensities relative to the $0-0$ peak appear to be underestimated in the simulated spectrum. The peaks from $19400 \mathrm{~cm}^{-1}$ to 
Table 2 Calculated bands of the absorption spectrum of perylene with a relative intensity of more than $10 \%$ of the $0-0$ peak. The transitions were assigned to the respective vibrational level of the excited state

\begin{tabular}{lcll}
\hline Wavenumber $\left(\mathrm{cm}^{-1}\right)$ & Shift $\left(\mathrm{cm}^{-1}\right)$ & Relative intensity & Assignment \\
\hline 20907 & 0 & 1.00 & \\
21265 & 358 & 0.43 & $1 \mathrm{a}_{\mathrm{g}}$ \\
21467 & 560 & 0.13 & $3 \mathrm{a}_{\mathrm{g}}$ \\
22229 & 1321 & 0.25 & $8 \mathrm{a}_{\mathrm{g}}$ \\
22346 & 1439 & 0.20 & $10 \mathrm{a}_{\mathrm{g}}$ \\
22557 & 1649 & 0.31 & $13 \mathrm{a}_{\mathrm{g}}$ \\
22586 & 1679 & 0.11 & $1 \mathrm{a}_{\mathrm{g}}+8 \mathrm{a}_{\mathrm{g}}$ \\
22914 & 2007 & 0.14 & $1 \mathrm{a}_{\mathrm{g}}+13 \mathrm{a}_{\mathrm{g}}$
\end{tabular}

$20200 \mathrm{~cm}^{-1}$ are shifted to higher energies compared to the corresponding experimental bands. There is a distinct peak in the experimental spectrum at $18800 \mathrm{~cm}^{-1}$, which is red-shifted compared to the $0-0$-band and has not been predicted in the calculated spectrum. A possible reason for these deviations from the experimental spectrum could be that the molecule occupies different sites within the neon matrix. This possibility has also been investigated by Halasinski et al. ${ }^{22}$ The different environment could result in a splitting of the peaks that is not predicted in the calculated spectrum of a free terrylene molecule. In spite of these discrepancies, there is good qualitative agreement between the simulated and the experimental spectrum. The calculated bands with a relative intensity of above $10 \%$ of the $0-0$ band are reported in Table 3 . The bands were assigned through comparison with the calculated vibrational spectrum of the excited state. Additionally, all bands with a relative intensity of above $2 \%$ of the $0-0$ peak are reported and assigned in the ESI. $\dagger$

There are larger differences between the calculated spectrum of the quaterrylene molecule and the experimental spectrum in Fig. 2c. The relative positions of the bands are well predicted by the simulated spectrum. The intensities of the calculated bands relative to the $0-0$-band are larger in the calculated spectrum. The calculations seem to overestimate the intensities of the higher energy vibronic transitions for the quaterrylene molecule. The higher intensity of the predicted vibronic transitions in conjunction with the applied peak broadening also leads to a higher baseline at the higher energy side of the spectrum. The presence of small red-shifted bands next to the $0-0$-band in the experimental spectrum could again be attributed to site-splitting effects ${ }^{22}$ in the neon matrix that have already been discussed for the terrylene molecule.

Table 3 Calculated bands of the absorption spectrum of terrylene with a relative intensity of more than $10 \%$ of the $0-0$ peak. The transitions were assigned to the respective vibrational level of the excited state

\begin{tabular}{lcll}
\hline Wavenumber $\left(\mathrm{cm}^{-1}\right)$ & Shift $\left(\mathrm{cm}^{-1}\right)$ & Relative intensity & Assignment \\
\hline 16035 & 0 & 1.00 & \\
16283 & 248 & 0.45 & $1 \mathrm{a}_{\mathrm{g}}$ \\
16532 & 497 & 0.10 & $2 \times 1 \mathrm{a}_{\mathrm{g}}$ \\
17355 & 1321 & 0.26 & $11 \mathrm{a}_{\mathrm{g}}$ \\
17460 & 1425 & 0.11 & $14 \mathrm{a}_{\mathrm{g}}$ \\
17604 & 1569 & 0.12 & $1 \mathrm{a}_{\mathrm{g}}+11 \mathrm{a}_{\mathrm{g}}$ \\
17682 & 1647 & 0.11 & $19 \mathrm{a}_{\mathrm{g}}$
\end{tabular}

Table 4 Calculated bands of the absorption spectrum of quaterrylene with a relative intensity of more than $10 \%$ of the $0-0$ peak. The transitions were assigned to the respective vibrational level of the excited state

\begin{tabular}{lcll}
\hline Wavenumber $\left(\mathrm{cm}^{-1}\right)$ & Shift $\left(\mathrm{cm}^{-1}\right)$ & Rel. intensity & Assignment \\
\hline 14821 & 0 & 1.00 & \\
14877 & 55 & 0.11 & $2 \times 1 \mathrm{a}_{\mathrm{u}}$ \\
15015 & 194 & 0.72 & $1 \mathrm{a}_{\mathrm{g}}$ \\
15209 & 388 & 0.26 & $2 \times 1 \mathrm{a}_{\mathrm{g}}$ \\
15401 & 579 & 0.12 & $5 \mathrm{a}_{\mathrm{g}}$ \\
16164 & 1342 & 0.50 & $14 \mathrm{a}_{\mathrm{g}}$ \\
16358 & 1536 & 0.37 & $1 \mathrm{a}_{\mathrm{g}}+14 \mathrm{a}_{\mathrm{g}}$ \\
16513 & 1692 & 0.12 & $25 \mathrm{a}_{\mathrm{g}}$ \\
16551 & 1730 & 0.15 & $2 \times 1 \mathrm{a}_{\mathrm{g}}+14 \mathrm{a}_{\mathrm{g}}$ \\
17506 & 2685 & 0.13 & $2 \times 14 \mathrm{a}_{\mathrm{g}}$ \\
& & &
\end{tabular}

The BH-LYP functional that was used for this calculation has $50 \%$ of HF exchange. This appears to reduce the shift necessary to compare the calculated spectrum to the experimental spectrum. This is not surprising since the TD-DFT method appears to underestimate adiabatic excitation energies while a functional with higher percentages of Hartree-Fock exchange overestimates the excitation energies as was shown in Table 1 , leading to possible error cancellation. The calculated bands with a relative intensity of above $10 \%$ of the $0-0$ band are reported in Table 4 . The bands were assigned through comparison with the calculated vibrational spectrum of the excited state. Additionally, all bands with a relative intensity of above $2 \%$ of the 0-0 peak are reported and assigned in the ESI. $\dagger$

Some of the low-frequency non-totally symmetric bands $\left(1 a_{u}, 2 a_{u}, 1 b_{2 g}, 1 b_{3 g}, 1 b_{1 u}\right.$ and $\left.8 b_{2 g}\right)$ from the excited state vibrational spectra contribute to the electronic absorption spectra of terrylene and quaterrylene as totally symmetric combination bands. These combination bands also combine with other totally symmetric bands as can be seen in the ESI. $\dagger$ The calculation of quantitatively correct bands in the absorption and emission spectra is difficult using TD-DFT as the prediction of the appropriate functional is crucial. TD-DFT also appears to underestimate the adiabatic excitation energies. This could be improved through usage of an ab initio method such as $\mathrm{CC} 2$ or $\mathrm{ADC}(2) .{ }^{49}$ The different scaling of the band intervals is introduced through the harmonic approximation and can only be alleviated through the calculation of anharmonic corrections to the vibrational frequencies. To achieve this the numerical calculation of higher order energy derivatives to construct high order force constant matrices is needed. Both of these measures are coupled to higher computational costs for large molecules.

\subsection{Fluorescence spectra}

The fluorescence spectra can be calculated from the ground and excited state energies at their respective equilibrium geometries and from the ground and excited state vibrational frequencies. These parameters were calculated for all three molecules using the appropriate functionals. Unfortunately experimental spectra are only available for the perylene molecule from Joblin et al. ${ }^{23}$ A lower lifetime parameter of $0.145 \mathrm{ps}$ was used to simulate the perylene spectra, because the spectral widths of these experimental spectra are larger. Both the 


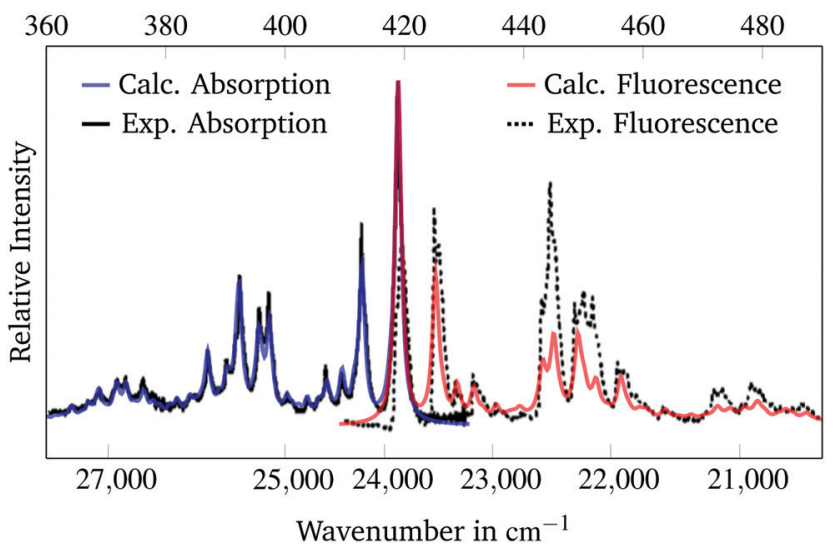

Fig. 3 Calculated and experimental ${ }^{23}$ absorption and fluorescence spectra of the $1 \mathrm{~B}_{3 \mathrm{u}}$ state of perylene. The simulated spectra are scaled and shifted to match the experimental spectra. A lifetime parameter of $0.145 \mathrm{ps}$ was used for broadening of the calculated spectra.

absorption and the fluorescence spectra are scaled by a factor of 0.98 and shifted by $3380 \mathrm{~cm}^{-1}$ analogously to Section 3.2 and the result is presented in Fig. 3.

As is to be expected from Section 3.2 there is high agreement between the experimental and the simulated absorption spectra. The simulated bands are able to reproduce the band structure of the experimental spectrum precisely. The position of the calculated fluorescence spectrum bands match those of the experimental spectrum. The spectral widths and heights of the bands are smaller than in the experimental spectrum. It appears as if the experimental fluorescence spectrum has larger spectral widths than the experimental absorption spectrum. Therefore the spectral widths and intensities could not be accurately reproduced by the calculated fluorescence spectrum. Overall, the position of the bands in the absorption and the fluorescence spectra are well predicted by the used methodology.

Fig. 4 presents the calculated absorption and emission spectra of all three molecules. No shift or scaling is applied in these figures. The lifetime parameters from Section 3.2 were used for all calculations.

The calculated spectra are very symmetrical. The emission band intensities are lower than the respective intensities of the absorption bands. The $0-0$-band has the highest intensity in all spectra. Therefore according to the Franck-Condon principle ${ }^{16,17}$ the predicted change in molecular geometry upon adiabatic electronic excitation is small which conforms with the rigid structure of the polyaromatic hydrocarbons. ${ }^{50}$ Fig. $4 \mathrm{c}$ clarifies why the baseline of the calculated spectrum in Fig. 2c appears to be raised on the high-energy side compared to the low-energy side of the spectrum. The calculated spectrum in Fig. 2c is cut off between two sets of overlapping vibronic transitions. The minimum between the transitions at around $18600 \mathrm{~cm}^{-1}$ is raised due to broadening of the neighbouring peaks. This effect is not observed in the experimental spectrum as the last set of bands is not visible due to lower experimental band intensities.

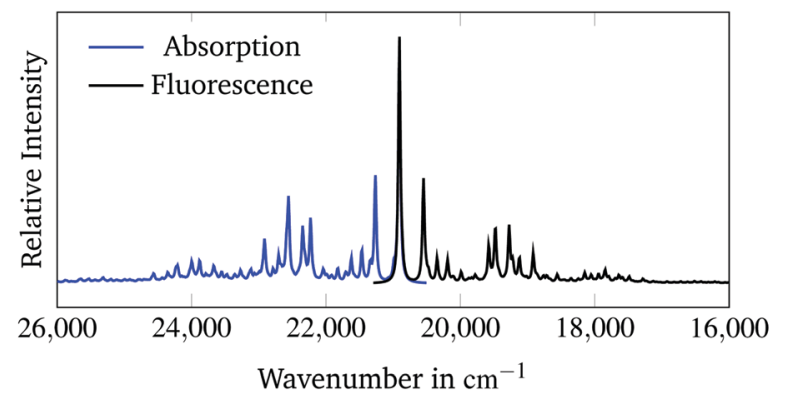

(a) Spectra of perylene, a lifetime parameter of $0.290 \mathrm{ps}$ was used for broadening.

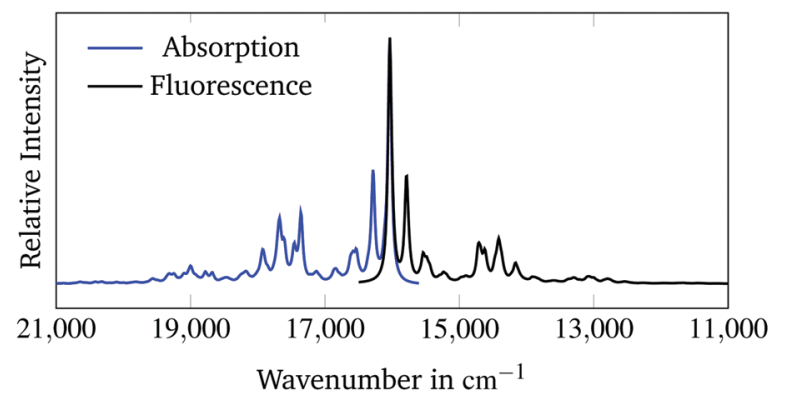

(b) Spectra of terrylene, a lifetime parameter of $0.169 \mathrm{ps}$ was used for broadening.

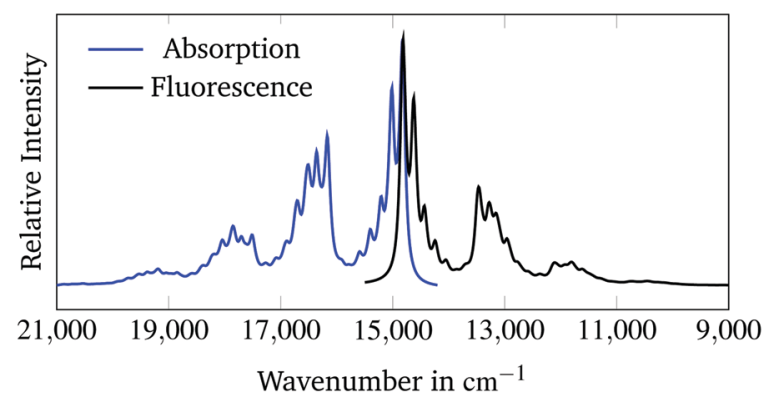

(c) Spectra of quaterrylene, a lifetime parameter of $0.097 \mathrm{ps}$ was used for broadening.

Fig. 4 Calculated absorption and fluorescence spectra of the $1 \mathrm{~B}_{3 \mathrm{u}}$ state of perylene, terrylene and quaterrylene.

\section{Conclusion}

The vertical excitation energies of three rylene molecules were calculated using TD-DFT. It was found that the percentage of exact Hartree-Fock exchange contained in the hybrid functional has a high effect on the accuracy of the calculated vertical excitation energies. There appears to be an optimal amount of Hartree-Fock exchange between $30 \%$ and $40 \%$ with a trend to higher percentages for larger polyaromatic molecules with smaller HOMO-LUMO gaps. These results were used to choose the optimal functional for the calculation of the absorption and emission spectra.

The simulated absorption spectra and their comparability to the experimental spectra of the rylenes validate the used methods and are a testament to their capability of describing the change in molecular geometry and molecular vibrations upon electronic excitation. Especially for perylene a high 
agreement between the simulation and the experiment was found. For the larger rylenes there were deviations from the experimental spectra. Some of these deviations are presumed to be a result of the experimental conditions of the matrixisolation spectroscopic experiment. The bands of the simulated spectra were assigned to vibronic transitions using the calculated excited state vibrational spectra.

The fluorescence spectra were calculated using the same methodology as the absorption spectra. It was found that the calculated fluorescence spectrum of perylene is in good agreement with the experimental spectrum. Unfortunately there are no comparison spectra for terrylene and quaterrylene available in the literature. The accuracy of the simulated fluorescence spectra could therefore not be assessed.

The generating function approach shows a lot of potential for the simulation of vibrationally resolved absorption and emission spectra of the polyaromatic hydrocarbons. The experimental spectra can be replicated with high accuracy which underlines that the consideration of Duschinsky effects ${ }^{19}$ within the Franck-Condon approximation ${ }^{16,17}$ is enough to accurately describe the studied molecules while HerzbergTeller vibronic coupling effects ${ }^{51}$ can be neglected. ${ }^{52}$ The only faults of the used methodology stem from the inability of TD-DFT to produce quantitatively accurate excitation energies and from the usage of the harmonic approximation to calculate molecular vibrations which makes shifting and scaling of the calculated spectra necessary for the qualitative comparison with the experiment. Conveniently this generating function technique is independent of the quantum chemical method used to calculate the required energies and could therefore be combined with more accurate procedures such as CC2 or $\operatorname{ADC}(2) .{ }^{49}$

\section{Conflicts of interest}

There are no conflicts to declare.

\section{Acknowledgements}

This work has been supported by the Academy of Finland through project 314821, the Swedish Cultural Foundation in Finland, and Magnus Ehrnrooth Foundation. J. G. thanks the ERASMUS+ SMP program for financial support. We thank Isaac Benkyi and Enrico Tapavicza for helpful discussions. The CSC the Finnish IT Center for Science and the Finnish Grid and Cloud Infrastructure (persistent identifier urn:nbn:fi:researchinfras-2016072533) are acknowledged for computer time.

\section{References}

1 C. Huang, S. Barlow and S. R. Marder, J. Org. Chem., 2011, 76, 2386-2407.

2 C. Li and H. Wonneberger, Adv. Mater., 2012, 24, 613-636.

3 E. Kozma and M. Catellani, Dyes Pigm., 2013, 98, 160-179.

4 M. Sadrai and G. R. Bird, Opt. Commun., 1984, 51, 62-64.
5 X. Zhan, A. Facchetti, S. Barlow, T. J. Marks, M. A. Ratner, M. R. Wasielewski and S. R. Marder, Adv. Mater., 2010, 23, 268-284.

6 L. Zhao, R. Lian, I. A. Shkrob, R. A. Crowell, S. Pommeret, E. L. Chronister, A. D. Liu and A. D. Trifunac, J. Phys. Chem. A, 2004, 108, 25-31.

7 A. M. Tokmachev, M. Boggio-Pasqua, D. Mendive-Tapia, M. J. Bearpark and M. A. Robb, J. Chem. Phys., 2010, 132, 044306.

8 T. P. Snow, Spectrochim. Acta, Part A, 2001, 57, 615-626.

9 T. P. Snow, V. L. Page, Y. Keheyan and V. M. Bierbaum, Nature, 1998, 391, 259-260.

10 L. J. Allamandola, G. G. M. Tielens and J. R. Barker, Astrophys. J., Suppl. Ser., 1989, 71, 733-775.

11 J. L. Puget and A. Léger, Annu. Rev. Astron. Astrophys., 1989, 27, 161-198.

12 D. M. Hudgins and L. J. Allamandola, Astrophys. J., 1999, 513, L69-L73.

13 F. Salama, E. L. O. Bakes, L. J. Allamandola and A. G. G. M. Tielens, Astrophys. J., 1996, 458, 621-636.

14 F. Salama, G. A. Galazutdinov, J. Krełowski, L. J. Allamandola and F. A. Musaev, Astrophys. J., 1999, 526, 265-273.

15 A. Omont, H. F. Bettinger and C. Tönshoff, Astron. Astrophys., 2019, 625, A41.

16 J. Franck and E. G. Dymond, Trans. Faraday Soc., 1926, 21, 536-542.

17 E. Condon, Phys. Rev., 1926, 28, 1182-1201.

18 M. Kasha, Discuss. Faraday Soc., 1950, 9, 14-19.

19 F. Duschinsky, Acta Physicochim. URSS, 1937, 7, 566.

20 E. Tapavicza, F. Furche and D. Sundholm, J. Chem. Theory Comput., 2016, 12, 5058-5066.

21 I. Benkyi, E. Tapavicza, H. Fliegl and D. Sundholm, Phys. Chem. Chem. Phys., 2019, 21, 21094-21103.

22 T. M. Halasinski, J. L. Weisman, R. Ruiterkamp, T. J. Lee, F. Salama and M. Head-Gordon, J. Phys. Chem. A, 2003, 107, 3660-3669.

23 C. Joblin, F. Salama and L. Allamandola, J. Chem. Phys., 1999, 110, 7287-7297.

24 M. Dierksen and S. Grimme, J. Phys. Chem. A, 2004, 108, 10225-10237.

25 P. H. P. Harbach and A. Dreuw, in Modeling of Molecular Properties, ed. P. Comba, Wiley VCH Verlag GmbH, 1st edn, 2011, ch. 3, pp. 29-47.

26 TURBOMOLE V7.4 2019, a development of University of Karlsruhe and Forschungszentrum Karlsruhe GmbH, 19892007, TURBOMOLE GmbH, since 2007; available from http://www.turbomole.com.

27 R. Ahlrichs, M. Bär, M. Häser, H. Horn and C. Kölmel, Chem. Phys. Lett., 1989, 162, 165-169.

28 F. Furche, R. Ahlrichs, C. Hättig, W. Klopper, M. Sierka and F. Weigend, WIREs Comput. Mol. Sci., 2013, 4, 91-100.

29 F. Weigend and R. Ahlrichs, Phys. Chem. Chem. Phys., 2005, 7, 3297-3305.

30 K. Eichkorn, F. Weigend, O. Treutler and R. Ahlrichs, Theor. Chem. Acc., 1997, 97, 119-124.

31 J. P. Perdew and Y. Wang, Phys. Rev. B: Condens. Matter Mater. Phys., 1992, 45, 13244-13249. 
32 J. Tao, J. P. Perdew, V. N. Staroverov and G. E. Scuseria, Phys. Rev. Lett., 2003, 91, 146401.

33 V. N. Staroverov, G. E. Scuseria, J. Tao and J. P. Perdew, J. Chem. Phys., 2003, 119, 12129-12137.

34 A. D. Becke, Phys. Rev. A: At., Mol., Opt. Phys., 1988, 38, 3098-3100.

35 C. Lee, W. Yang and R. G. Parr, Phys. Rev. B: Condens. Matter Mater. Phys., 1988, 37, 785-789.

36 A. D. Becke, J. Chem. Phys., 1993, 98, 5648-5652.

37 J. P. Perdew, M. Ernzerhof and K. Burke, J. Chem. Phys., 1996, 105, 9982-9985.

38 J. P. Perdew, K. Burke and M. Ernzerhof, Phys. Rev. Lett., 1996, 77, 3865-3868.

39 A. D. Becke, J. Chem. Phys., 1993, 98, 1372-1377.

40 O. Treutler and R. Ahlrichs, J. Chem. Phys., 1995, 102, 346-354.

41 M. Casida and M. Huix-Rotllant, Annu. Rev. Phys. Chem., 2012, 63, 287-323.

42 R. Bauernschmitt and R. Ahlrichs, Chem. Phys. Lett., 1996, 256, 454-464.
43 D. R. F. Furche, in Theoretical and Computational Chemistry, ed. M. Olivucci, Elsevier Science, 2005, vol. 16, ch. III.

44 F. Furche and R. Ahlrichs, J. Chem. Phys., 2002, 117, 7433-7447.

45 Avogadro: an open-source molecular builder and visualization tool, Version 1.2.0, 2017, https://protect-eu.mimecast.com/s/ GfgpCzvwXtJ9j5c4kqhf?domain=avogadro.cc.

46 M. D. Hanwell, D. E. Curtis, D. C. Lonie, T. Vandermeersch, E. Zurek and G. R. Hutchison, J. Cheminf., 2012, 4, 17.

47 P. Deglmann, F. Furche and R. Ahlrichs, Chem. Phys. Lett., 2002, 362, 511-518.

48 P. Deglmann and F. Furche, J. Chem. Phys., 2002, 117, 9535-9538.

49 C. Hättig, Advances in Quantum Chemistry, Elsevier, 2005, pp. 37-60.

50 R. Rieger and K. Müllen, J. Phys. Org. Chem., 2010, 315-325.

51 G. Herzberg and E. Teller, Z. Phys. Chem., Abt. B, 1933, 21, 410-446.

52 F. Santoro, A. Lami, R. Improta, J. Bloino and V. Barone, J. Chem. Phys., 2008, 128, 224311. 